\title{
Folic acid use in pregnant patients presenting to the emergency department
}

\author{
Jacob Steenblik, Erika Schroeder', Burke Hatch', Steven Groke', Camille Broadwater-Hollifield ', Michael Mallin', \\ Matthew Ahern ${ }^{1}$ and Troy Madsen ${ }^{1,2^{*}}$
}

\begin{abstract}
Background: The US Preventive Services Task Force has recommended daily folic acid supplementation for women planning on becoming pregnant in an effort to prevent fetal neural tube defects. We evaluated pregnant patients presenting to the emergency department to determine rates of folic acid supplementation.

Methods: We surveyed a convenience sample of pregnant patients who presented to the University of Utah Emergency Department (ED) between 1 January 2008, and 30 April 2009, regarding pregnancy history and prior medical care.

Results: One hundred thirty-five patients participated in the study. Eighty-four patients (62.2\%) reported current folic acid supplementation. Sixty-six patients identified themselves as Caucasian and 69 as non-Caucasian race. There was a significant difference in folic acid use between Caucasian and non-Caucasian women $(p=0.035)$. The majority of Caucasian women (71.2\%) reported daily folic acid use versus approximately one-half of non-Caucasian women (53.6\%). Both groups were similar in accessing a primary care provider (PCP) for pregnancy care prior to the ED visit (53\% vs. $49.3 \%, p=0.663)$, and rates of folic acid use were similar in those who had seen a PCP $(85.7 \%$ vs. $76.5 \%, p=0.326)$. Language did not have a significant association with folic acid use.

Conclusion: A large percentage of pregnant ED patients did not report current folic use, and there was a significant difference between Caucasian and non-Caucasian women in rates of folic acid supplementation. This study highlights the potential role of the ED in screening patients for folic acid supplementation.
\end{abstract}

\section{Introduction}

In the United States, approximately one in every 1,000 pregnancies is affected by a neural tube defect (NTD) [1]. Among the most common types of NTDs, spina bifida and anencephaly are estimated to affect approximately 3,000 pregnancies each year in the US [2-7]. In an effort to curtail these preventable birth defects, the Food and Drug Administration (FDA) has suggested that women of childbearing age consume a minimum of $400 \mu \mathrm{g}(0.4 \mathrm{mg})$ of folic acid daily [1,2,8-10]. Despite these recommendations, total folate consumption remains well below the recommended levels in Hispanic communities when compared to non-Hispanic communities $[1-5,8,10]$. Additional research has suggested that

\footnotetext{
* Correspondence: troy.madsen@hsc.utah.edu

${ }^{1}$ University of Utah, Salt Lake City, UT, USA

Full list of author information is available at the end of the article
}

non-Caucasian females of child-bearing age are significantly less likely to take a prenatal vitamin [10].

Pregnant patients presenting to the emergency department (ED) may represent a higher risk group that is less likely to have received prenatal care or appropriate education regarding folic acid supplementation [11]. Emergency departments have been successful in performing nursing screening and intervention for domestic violence prevention and alcohol abuse, and may represent an appropriate setting for intervention regarding folic acid supplementation in pregnancy $[12,13]$.

We sought to evaluate the rates of folic acid use among pregnant patients presenting to the ED. Furthermore, we intended to identify patient characteristics related to folic acid use in an attempt to potentially define the role of the ED in screening for folic acid use and aiding in the prevention of neural tube defects.

\section{SpringerOpen ${ }^{\odot}$}

(C) 2011 Steenblik et al; licensee Springer. This is an Open Access article distributed under the terms of the Creative Commons Attribution License (http://creativecommons.org/licenses/by/2.0), which permits unrestricted use, distribution, and reproduction in any medium, provided the original work is properly cited. 


\section{Methods}

We conducted a survey study of a convenience sample of pregnant ED patients over the 16-month period from 1 January 2008, through 30 April 2009 at the University of Utah Medical Center ED in Salt Lake City, Utah. The University of Utah ED is an urban, academic ED that treats approximately 39,000 patients per year.

All pregnant patients in the ED were eligible to participate. Patients were approached by trained research associates and asked to complete a survey regarding their pregnancy history and current medications. All pregnant patients who participated in the study had presented with pregnancy-related complaints of abdominal pain and/or vaginal bleeding. Research associates were present in the ED 7 days per week from 8 a.m. until midnight.

The survey consisted of questions regarding pregnancy history, current medications, and prenatal care. Patients were specifically asked the question, "Are you taking prenatal vitamins?" We utilized this question as we felt the term "prenatal vitamins" would be the term most familiar to patients to describe a folic acid-containing supplement.

Patients were asked to self-identify their race and primary language with free-text spaces in which the patient recorded this information. Utilizing self-reported race, patients were grouped according to reported race using United State Census classifications [14]. In evaluating rates of folic acid use, comparisons were made between patients reporting Caucasian race and those reporting a race that was not Caucasian (non-Caucasian). In two cases, patients reported "multi-racial" as their selfreported race. For statistical analysis, these patients were classified as non-Caucasian.

Patients were asked about their medical care during the pregnancy with the following question: "Have you been to a health care professional (OB, family practitioner, midwife) for care during this pregnancy?" Patients were asked to report the date of the first day of their last menstrual period, and gestational age was calculated based on this. The survey was one that we developed and that underwent internal revision and validation without an external validation processes (see Appendix).

Chi-square testing and Fisher's exact test were used for categorical variables (SPSS v. 16.0). For associations, we report $p$-value, odds ratios, and $95 \%$ confidence intervals (CI). This study received approval from the University of Utah Institutional Review Board (IRB).

\section{Results}

One hundred thirty-five pregnant women participated in the study during the 16 -month period. Of these, 23 patients $(17 \%)$ presented with vaginal bleeding, 44 patients $(32.6 \%)$ presented with abdominal pain, and 68 patients (50.1\%) presented with both vaginal bleeding and abdominal pain. The average age of the patients was 25.1 years (range 16-42 years). The average number of pregnancies per patient was 2.8 (range 1-9) with an average of 1.1 previous live births per patient (range 06). The average estimated gestational age was 73.2 days (range 13-147 days) (see Table 1).

Of the women, $62.2 \%$ reported current use of a prenatal vitamin at the time of the ED visit. We noted a significant difference in prenatal vitamin use between patients who identified themselves as Caucasian and those who self-identified as a non-Caucasian race. Sixtysix patients (48.9\%) identified themselves as Caucasian, while 69 patients $(51.1 \%)$ identified themselves as of non-Caucasian race. Patients who self-reported a nonCaucasian race identified themselves as follows: Hispanic (68.1\%), African American (11.6\%), Native American (7.2\%), Pacific Islander (5.8\%), Asian (4.3\%), and multiracial $(2.9 \%)$; $71.2 \%$ of Caucasian women reported prenatal vitamin use compared to $53.6 \%$ of non-Caucasian women $(p=0.035$, OR $=2.14,1.05-4.36)$ (see Figure 1 )

Caucasian and non-Caucasian patients were similar in reporting having seeing a primary care provider for prenatal care prior to the ED visit ( $53 \%$ vs. $49.3 \%, p=0.663$, $\mathrm{OR}=1.16,0.59-2.28)$. Among those who were seen by a primary care provider $(n=69)$, rates of prenatal vitamin use were similar (Caucasians: $85.7 \%$ vs. non-Caucasians: $76.5 \%, p=0.326$, OR $=1.85,0.54-6.35$ ). Language did not have a significant association with parental vitamin use. Of the patients, $25.9 \%$ identified their primary language as a language other than English. Sixty-six percent of patients who stated their primary language was English reported prenatal vitamin use vs. $51.4 \%$ who identified their primary language as a language other than English $(p=0.126$, OR $=1.83,0.84-4.01)$.

\section{Discussion}

Approximately $70 \%$ of neural tube defects could be prevented with the consumption of folic acid before conception and in the early stages of pregnancy [5,8-10]. As we have reported in our study, a significant percentage

\section{Table 1 Patient Characteristics}

\begin{tabular}{ll}
\hline Characteristic & Number/Percentage (Range) \\
\hline Total Patients & 135 \\
\hline Average Age & 25.1 years (16-42) \\
\hline Number of Pregnancies & $2.8(1-9)$ \\
\hline Previous Live Births & $1.1(0-6)$ \\
\hline Average Gestational Age & 73.2 days (13-147) \\
\hline Non-Caucasian Race & $51.10 \%$ \\
\hline Current Prenatal Vitamin Use & $62 \%$ \\
\hline
\end{tabular}




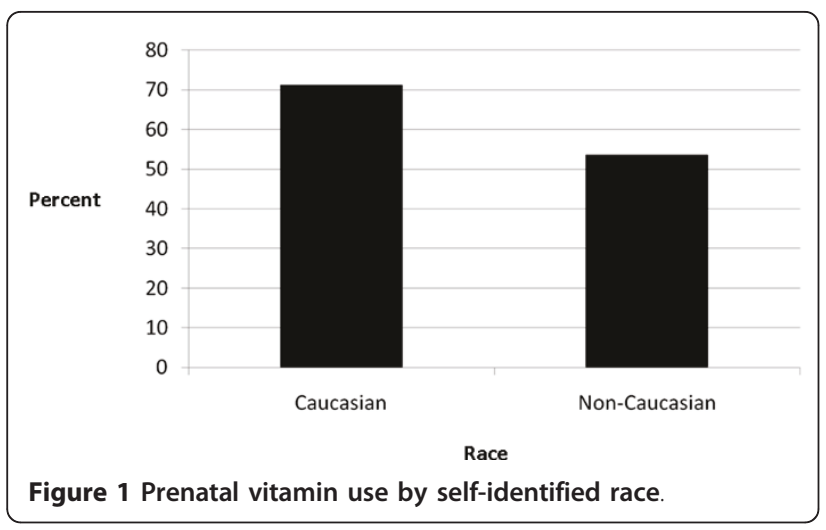

of pregnant patients presenting to an emergency department did not report current folic acid supplementation. Perhaps even more notable, this difference was particularly pronounced when comparing patients who selfidentified as a non-Caucasian race. Previous studies have noted a more significant dietary deficiency of folic acid in non-Caucasian patients $[1,5,8,10]$.

The reason for lower rates of folic acid supplementation in non-Caucasian patients in our study does not seem to be related to language barriers leading to misunderstanding or failure to receive appropriate guidelines for folic acid supplementation. We found that those who identified a primary language other than English were not significantly less likely to reports folic acid supplementation. This difference also did not seem to be related to discrepancies in the prenatal care provided between Caucasian and non-Caucasian patients who saw a physician prior to their ED visit. Of those who had reported visiting a health care provider prior to their ED visit, folic acid supplementation was similar between these two groups.

Given these findings, this study suggests a potential role for the ED in the prevention of NTDs. As this study demonstrates, a high percentage of pregnant patients are not currently using folic acid supplementation. Folic acid supplementation education delivered in the ED may be a viable public health initiative. This intervention could be as simple as screening childbearing-age women for folic acid use and providing an information sheet on the benefits of folic acid supplementation, as well as a list of community resources available to these patients.

While our study suggests the potential for a folic acid intervention program, it does not suggest that such an intervention would be effective in improving the rates of folic acid supplementation among pregnant women. Other ED-based intervention programs, however, have previously proven effective. Examples of these include screening for domestic violence and alcohol abuse
$[12,13]$. Further research would seem indicated to determine the efficacy of an ED-based folic acid supplementation intervention.

\section{Limitations}

This study did not evaluate the outcomes of the study participants to determine pregnancy complications for those who did not report folic acid use. For many of the patients we evaluated, it is likely that initiation of folic acid at the time of their visit may have been too late to prevent neural tube defects, given that the range of reported gestational age extended to 147 days.

As a convenience sample of patients, this does not represent all pregnant ED patients during the study period, and thus may not adequately represent folic acid use in this population. The convenience sample is subject to bias based on both the availability of research associates as well as patient willingness to participate in the survey. Thus, this may not adequately represent folic acid use among the full spectrum of pregnant ED patients.

Similarly, our study focused only on those patients who presented to the ED with pregnancy-related symptoms. Ideally, folic acid supplementation would be initiated by all women of childbearing age. Although we presume that the rate of folic acid use in this larger population would be even less than in the pregnant patients we studied, we are unable to draw conclusions related to this population from the information we have gathered.

It is unclear whether low rates of folic acid supplementation may have been unique to our catchment area, or whether these lower rates, particularly among non-Caucasian patients, may have been due to characteristics of the primary care network or health care initiatives in our region. Similarly, while we did not find that language was significantly associated with differences in folic acid supplementation, language barriers or cultural differences between patient and physician may affect the efficacy of physician counseling and recommendations. We have not specifically evaluated the multiple questions related to the provider-patient dynamic and how this may have affected the results that we noted in our study.

\section{Conclusion}

Neural tube defects may be prevented through folic acid supplementation in early pregnancy. Our study demonstrated both a low rate of folic acid supplementation among pregnant ED patients and a significantly lower rate of folic acid use among non-Caucasian patients. This study suggests the potential need for an ED-based educational intervention program as a means to improve folic acid supplementation in pregnancy. 


\section{Appendix}

\section{Emergency Department Pregnancy Study Questionnaire Please tell us about yourself:}

Name:

Telephone number:

E-mail address or alternative phone number:

Primary language:

Age:

Race:

Please tell us about your previous pregnancy

\section{history:}

How many times have you been pregnant?

How many times have you given birth?

How many times have you had live births?

How many spontaneous failed pregnancies or miscarriages have you experienced?

How many induced abortions have you experienced?

How many Cesarean deliveries have you experienced?

Have you ever been hospitalized for treatment of pelvic inflammatory disease? Yes No

Have you ever been treated as an outpatient (out of the hospital) for a pelvic infection from chlamydia or gonorrhea? Yes No

Have you ever used an intrauterine device (IUD) for birth control? Yes No

Have you ever had pelvic surgery (not including cesarean section)? Yes No

Please tell us about the symptoms you have experienced recently:

What was the date of your last menstrual period?

Have you had an ultrasound to evaluate your pregnancy prior to this visit? Yes No

If so, what is your estimated delivery date based on this ultrasound?

Have you been to a health care professional (OB, family practitioner, midwife) for care during this pregnancy? Yes No

Are you taking prenatal vitamins? Yes No

Are you taking aspirin? Yes No

Do you smoke? Yes No

Please list any other medications you are currently taking:

How much pain are you experiencing? (please circle one of the choices below)

None (no pain)

Mild (less than your menstrual period)

Moderate (equal to your menstrual period)

Severe (more than your menstrual period)

How much vaginal bleeding are you experiencing? (please circle one of the choices below)

None (no bleeding)

Mild (less than your menstrual period)

Moderate (equal to your menstrual period)

\section{Thank you for your participation in this study.}

\section{Author details}

${ }^{1}$ University of Utah, Salt Lake City, UT, USA ${ }^{2}$ Division of Emergency Medicine, University of Utah, 30 N. 1900 E. 1C26, Salt Lake City, UT 84098, USA

\section{Authors' contributions}

$\mathrm{TM}, \mathrm{ES}, \mathrm{BH}$ designed the study. TM, JS, ES, BH, MM, CBH, and MA contributed to the data analysis and review. TM, JS, ES, BH, MM, CBH, and MA provided significant contribution in the writing and revision of the manuscript.

\section{Competing interests}

The authors declare that they have no competing interests.

Received: 7 February 2011 Accepted: 24 June 2011

Published: 24 June 2011

\section{References}

1. Yang QH, Carter HK, Mulinare J, Berry RJ, Friedman JM, Erickson JD: Raceethnicity differences in folic acid intake in women of childbearing age in the United States after folic acid fortification: findings from the National Health and Nutrition Examination Survey, 2001-2002. Am J Clin Nutr 2007, 85(5):1409-16.

2. Quinlivan EP, Gregory JF: Effect of food fortification on folic acid intake in the United States. Am J Clin Nutr 2003, 77(1):221-5.

3. Bodnar LM, Himes KP, Venkataramanan R, Chen JY, Evans RW, Meyer JL, Simhan HN: Maternal serum folate species in early pregnancy and risk of preterm birth. Am J Clin Nutr 2010, 92(4):864-71.

4. Bentley TG, Willett WC, Weinstein MC, Kuntz KM: Population-level changes in folate intake by age, gender, and race/ethnicity after folic acid fortification. Am J Public Health 2006, 96(11):2040-7.

5. US Preventive Services Task Force: Folic acid for the prevention of neural tube defects: US Preventive Services Task Force recommendation statement. Ann Intern Med 2009, 150(9):626-31.

6. Vahratian A, Siega-Riz AM, Savitz DA, Thorp JM Jr: Multivitamin use and the risk of preterm birth. Am J Epidemiol 2004, 160(9):886-92.

7. Bailey RL, Dodd KW, Gahche JJ, Dwyer JT, McDowell MA, Yetley EA, Sempos CA, Burt VL, Radimer KL, Picciano MF: Total folate and folic acid intake from foods and dietary supplements in the United States: 20032006. Am J Clin Nutr 2010, 91(1):231-7.

8. Hamner $\mathrm{HC}$, Mulinare J, Cogswell ME, Flores AL, Boyle CA, Prue CE, Wang CY, Carriquiry AL, Devine O: Predicted contribution of folic acid fortification of corn masa flour to the usual folic acid intake for the US population: National Health and Nutrition Examination Survey 20012004. Am J Clin Nutr 2009, 89(1):305-15.

9. Rosenberg KD, Gelow JM, Sandoval AP: Pregnancy intendedness and the use of periconceptional folic acid. Pediatrics 2003, 111(5 Part 2):1142-5.

10. Williams LJ, Rasmussen SA, Flores A, Kirby RS, Edmonds LD: Decline in the prevalence of spina bifida and anencephaly by race/ethnicity: 19952002. Pediatrics 2005, 116(3):580-6.

11. Brunette DD, Sterner SP: Prehospital and emergency department delivery: a review of eight years experience. Ann Emerg Med 1989, 18(10):1116-8.

12. Schafer SD, Drach LL, Hedberg K, Kohn MA: Using diagnostic codes to screen for intimate partner violence in Oregon emergency departments and hospitals. Public Health Rep 2008, 123(5):628-35.

13. Stewart SH, Doscher A, Miles S, Borg KT: Identification and riskstratification of problem alcohol drinkers with minor trauma in the emergency department. West J Emerg Med 2010, 11(2):133-7.

14. U.S Census Bureau: Racial and ethnic classifications used in Census 2000 and beyond.[http://www.census.gov/population/www/socdemo/race/ racefactcb.html], Accessed May 19, 2011..

doi:10.1186/1865-1380-4-38

Cite this article as: Steenblik et al:: Folic acid use in pregnant patients presenting to the emergency department. International Journal of Emergency Medicine 2011 4:38. 